\title{
Thrombolytic Therapy for Acute Pulmonary Embolism
}

\author{
Victor F. Tapson, MD ${ }^{1}$ \\ ${ }^{1}$ Department of Medicine, Center for Pulmonary Vascular Disease, \\ Duke University Medical Center, Durham, North Carolina \\ Semin Thromb Hemost 2013;39:452-458.
}

\begin{abstract}
Address for correspondence Victor F. Tapson, MD, Department of Medicine, Center for Pulmonary Vascular Disease, 128 Hanes House, 330 Trent Drive, Duke University Medical Center, Durham, NC 27710 (e-mail: tapso001@mc.duke.edu).
\end{abstract}

\author{
Abstract \\ Keywords \\ - pulmonary embolism \\ - deep venous \\ thrombosis \\ - thrombolytic therapy \\ - right ventricular \\ function \\ - catheter-based \\ embolectomy
}

Thrombolytic therapy accelerates the dissolution of acute pulmonary embolism and is potentially lifesaving. The goal of this article is to offer a critical analysis of the use of thrombolytic therapy in this setting. Guidelines have been written and modified and new ones have been published over the past several years. Although an evidence base exists, unanswered questions remain. Despite the potential benefit of rapid clot lysis, nonpathologic thrombi are also lysed, so that thrombolytic therapy can cause significant bleeding complications. Massive acute pulmonary embolism is the clearest indication for these drugs, and although thrombolysis has been studied in submassive pulmonary embolism, this scenario remains more controversial. Traditionally, thrombolytic agents have been delivered intravenously, but intraembolic therapy via a pulmonary artery catheter has gained momentum. Few randomized trials have been conducted, however. Only three agents have been approved for use in the United States: streptokinase, urokinase, and tissue-type plasminogen activator. Urokinase is not currently available for use in the United States. The latter agent has been most widely used on the basis of proven benefit with a relatively short (2-hour) infusion. Newer, unapproved agents include tenecteplase and reteplase. Risk stratification in acute pulmonary embolism is important in determining which patients are the most appropriate candidates for thrombolysis, with careful consideration of contraindications.
Pulmonary embolism (PE) is a common, life-threatening clinical entity encountered by clinicians of all specialties. In spite of decades of clinical trials, only a limited number of randomized controlled trials have compared thrombolytic therapy with conventional anticoagulation in the treatment of PE. ${ }^{1-11}$ These trials together account for less than 900 patients. $^{1-3,5-11}$ Furthermore, there have not been large enough studies that include the patients most likely to benefit. In contrast, thrombolytic therapy had been studied in many thousands of patients with acute myocardial infarction before its acceptance into standard clinical practice. ${ }^{12}$ Thus, controversies still remain with regard to the precise indications in acute PE. We present background data, controversies, and general approaches to the use of thrombolytic therapy in acute PE.

Although our focus is thrombolysis, it should be emphasized that anticoagulation clearly improves mortality and should be instituted promptly when the clinical suspicion is high or the diagnosis has already been made, with careful attention to contraindications. Risk assessment and therapeutic decisions should be made as quickly as possible because the reported mortality rate without treatment is approximately $30 \%$ compared with approximately 4 to $8 \%$ when treated. ${ }^{13-16}$

Standard therapy for acute PE includes therapeutic anticoagulation with weight-adjusted subcutaneous low-molecular-weight heparin or fondaparinux. Renal function must be considered with the latter two drugs. ${ }^{13}$ In November 2012, oral rivaroxaban was approved for use in the United States for acute deep venous thrombosis (DVT) and acute PE, and its use will likely increase significantly. Importantly, anticoagulation only helps to prevent further thrombus formation and cannot itself dissolve thromboemboli that are already present; published online March 28, 2013
Issue Theme Biologic Role of the Plasminogen-Plasmin System: Thrombolysis, Bleeding, and Beyond; Guest Editors, Hau C. Kwaan, MD, FRCP, and Andrew P. Mazar, PhD.
Copyright (c) 2013 by Thieme Medical Publishers, Inc., 333 Seventh Avenue, New York, NY 10001, USA. Tel: +1(212) 584-4662.
DOI http://dx.doi.org/ $10.1055 / \mathrm{s}-0033-1334145$ ISSN 0094-6176. 
therefore, in certain circumstances more aggressive therapy is needed. Finally, when anticoagulation is contraindicated, inferior venacaval filter placement should be undertaken. ${ }^{13}$

A crucial issue in acute PE is how to risk stratify patients; that is, how to translate the overall severity of the PE event into a meaningful treatment plan. Although clinical algorithms are useful in stratification, the clinical severity of acute PE is highly variable, requiring careful evaluation on a caseby-case basis. The potential complications from thrombolytic therapy adds another level of complexity to risk stratification.

When acute PE is proven or highly suspected and anticoagulation is initiated, more aggressive strategies should be considered, including thrombolytic therapy. Risk stratification involves a global assessment of the patient with a focus on right ventricular (RV) function. Massive PE (associated with hemodynamic instability) is generally a more straightforward decision.

\section{Massive Pulmonary Embolism}

In the patient with proven PE and clear hemodynamic instability and in the absence of absolute contraindications, most clinicians will agree to initiate systemic thrombolytics. ${ }^{13,14}$ However, the precise definition of hemodynamic instability and thus massive PE is not always clear. Hemodynamic instability has generally been defined as a systolic blood pressure less than $90 \mathrm{~mm} \mathrm{Hg} .{ }^{13}$ A sustained drop in blood pressure to less than $90 \mathrm{~mm} \mathrm{Hg}$ for at least 15 minutes has also been used. $^{14}$ The American College of Chest Physicians guidelines (February 2012) state that "in patients with acute PE associated with hypotension (eg, systolic BP $<90 \mathrm{~mm} \mathrm{Hg}$ ) who do not have a high bleeding risk, we suggest systemically administered thrombolytic therapy over no such therapy (Grade 2C)."13 Some individuals normally run a low systolic blood pressure, so other clinical parameters must be considered. On the contrary, a substantial drop in pressure, even if it remains above $90 \mathrm{~mm} \mathrm{Hg}$ systolic, should be taken seriously. A patient with syncope at rest and proven PE may have an extensive clot burden by computed tomographic angiography but not remain hypotensive when assessed. Such patients require careful assessment with regard to their hemodynamic status. Other parameters (see Risk Stratification and Submassive PE, below) should be considered when questions remain after blood pressure has been determined and/or monitored.

Thus, the cases rarely stimulating disagreement are those in which the patient has symptomatic hypotension requiring hemodynamic support. Although clinical experience supports this approach, no clinical study has demonstrated unequivocally even in this setting that thrombolytics improve mortality over anticoagulation alone. ${ }^{13-17}$ Nonetheless, given the high mortality rate of this subgroup, few clinical researchers could comfortably randomize such patients.

\section{Risk Stratification and Submassive Pulmonary Embolism}

Risk stratification has been recommended in clinical guidelines, and the approach continues to evolve. The management of either submassive PE (hemodynamically stable but with abnormal RV function) or cases of extensive clot burden/ saddle embolus with minimal or no demonstrable RV dysfunction continue to be hotly debated. The Pulmonary Embolism Severity Index has been studied and simplified. ${ }^{18}$ Although it can predict outcomes, it appears most useful for identifying patients at low risk who could be discharged early or managed entirely in the outpatient setting. It cannot be used to estimate the potential impact of therapy on outcomes. The focus has to be specific clinical parameters such as RV function.

The clinical approach includes a global assessment of the patient, including vital signs, presence/degree of RV dysfunction, extent of emboli by computed tomography or ventilation perfusion scan, biomarkers, oxygenation, and residual DVT. ${ }^{14}$ Parameters reflecting severity that should be considered are listed in -Table 1. Potential contraindications to thrombolytics (see below), associated comorbidities, center expertise, and patient preference must be taken into consideration.

Although several parameters should be examined, a key focus is how well RV function is being maintained. Echocardiography, particularly performed by an expert operator, can provide detailed information. The RV status correlates directly with cardiogenic shock and, hence, mortality. ${ }^{16,17,19}$ Accurate, detailed assessment of RV function is not yet perfected, but extremes of RV size and function may be useful in decision making. It is feasible that different measures of RV size or function are associated with different prognoses. Several tools have been proposed thus far. Findings that raise significant concern include a significantly enlarged and/or hypokinetic RV with an interventricular septum that compromises

Table 1 Considerations in risk stratification

\begin{tabular}{|l|}
\hline Clinical status of the patient \\
General appearance (acutely ill, confused, etc.) \\
Hypotension (relative or absolute) \\
Tachycardia/tachypnea \\
\hline Electrocardiography \\
Tachycardia \\
RV strain \\
\hline Echocardiography \\
RV enlargement/hypokinesis \\
Right atrial enlargement \\
Clot-in-transit \\
Patent foramen ovale or other shunt \\
\hline Computed tomographic angiography \\
RV enlargement \\
Embolic burden/proximal extent of emboli \\
Presence/extent of venous thrombosis (if CTV is done) \\
\hline Compression ultrasound \\
Presence/extent of venous thrombosis \\
\hline Biomarkers \\
Serum troponin \\
Brain natriuretic peptide \\
\hline Oxygenationa
\end{tabular}

Abbreviation: CTV, computed tomographic venography. ${ }^{a}$ Although severity of hypoxemia has been less well-studied in acute PE as an independent predictor of mortality, it logically should be considered in risk stratification. 
filling of the left ventricle, potentially leading to systemic hypotension. ${ }^{14}$ One rapid technique is to simply measure the chamber proportions by computed tomographic angiography; an increased right-to-left ventricle ratio $\geq 1$ suggests RV dysfunction and has been shown to predict mortality. ${ }^{20,21}$

Biomarkers are being routinely used for risk stratification. Elevated levels of brain natriuretic peptide, pro-brain natriuretic peptide, and cardiac troponins (both $\mathrm{T}$ and $\mathrm{I}$ ) have been shown to correlate with RV compromise.22-24 A metaanalysis of 1,985 PE patients from 20 clinical studies revealed that any elevation of the troponin level (microinfarction) confers a fivefold increase in short-term mortality. ${ }^{23}$ Troponin levels appear to predict outcome not only for PE patients in shock but also for those patients who are hemodynamically stable at presentation. ${ }^{23}$

The introduction of highly sensitive troponin assays appears to have improved their diagnostic sensitivity. ${ }^{25}$ Preliminary findings have suggested that a highly sensitive troponin $\mathrm{T}$ cutoff value of $14 \mathrm{pg} / \mathrm{mL}$ may be associated with a high prognostic sensitivity and negative predictive value for an adverse 30-day outcome after acute $P E^{26}$ Although these findings may indicate that the novel highly sensitive troponin $\mathrm{T}$ assay might be helpful for identifying patients appropriate for early discharge, they might be expected to lower the specificity for detecting severe PE cases. In fact, however, when combined with clinical risk stratification, they may improve the ability to predict poor outcomes. ${ }^{27}$

Echocardiography and troponin data have been examined together. Jiménez and colleagues reported data on 591 normotensive patients diagnosed with PE who were examined with echocardiography or troponin testing and compression ultrasound of the legs. ${ }^{28}$ The primary outcome of PErelated death within 30 days occurred in 37 patients $(6.3 \%$; 95\% confidence interval [CI], 4.3 to $8.2 \%$ ). Patients with both RV dysfunction and concomitant DVT had a PE-related mortality of $19.6 \%$, compared with $17.1 \%$ of patients with elevated troponin I and concomitant DVT and $15.2 \%$ of patients with an elevated troponin I and RV dysfunction. The use of any twotest strategy had a higher specificity and positive predictive value compared with the use of any test by itself. The combination of echocardiography or troponin testing and compression ultrasound of the legs improved prognostication compared with the use of any test by itself for the identification of those at high risk of PE-related death. ${ }^{28}$ These data help to get at the core of the crucial debate of who should receive thrombolytic therapy.

Residual clot burden in the legs has also been examined in the international multicenter Registro Informatizado de la Enfermedad Tromboembolica. ${ }^{29}$ These data suggest that patients with acute PE who have concomitant residual DVT have a higher mortality. In an external validation cohort of 4,476 patients with acute PE enrolled in this registry, concomitant DVT remained a significant predictor of all-cause (adjusted hazard ratio, 1.66; 95\% CI, 1.28 to $2.15 ; p<0.001$ ) and PEspecific mortality (adjusted hazard ratio, 2.01; 95\% CI, 1.18 to $3.44 ; p=0.01) .{ }^{29}$ Although data are not from a prospective randomized trial, they are logical and compelling.
Finally, extent of clot burden in the lungs has been studied. PE extent has been calculated by the Qanadli obstruction index, in which the highest score is 40 , and represents complete obstruction of the main pulmonary artery. ${ }^{21}$ In one large nonrandomized study of 579 patients, mean obstruction index values were similar in patients who died and/or had clinical deterioration at 30 days compared with patients who had a favorable prognosis. ${ }^{21}$ However, central location of emboli was a predictor of all cause death and/or clinical deterioration in patients with acute PE who were hemodynamically stable. ${ }^{21}$ It is quite logical that clot burden correlates with mortality in acute PE; it must. But predicting mortality based on this parameter alone is difficult. - Fig. 1 demonstrates a large PE; based on the size of this embolus, thrombolytic therapy would be considered, but other parameters of severity would also be examined.

Few large, randomized thrombolytic trials have been conducted in submassive PE. In a large prospective, randomized trial, Konstantinides et al demonstrated that patients who received tissue plasminogen activator (tPA) were significantly less likely to deteriorate clinically than those who received placebo (11 vs. $25 \%) .{ }^{30}$ No mortality difference was demonstrated, but there was a higher rate of rescue thrombolysis in the placebo group. Several smaller studies have been published. It is anticipated that more definitive evidence will be available after the conclusion of the international multicenter Pulmonary Embolism THrOmbolysis (PEITHO) study, which compares thrombolysis with tenecteplase plus anticoagulation versus anticoagulation alone in this subgroup of patients. ${ }^{31}$

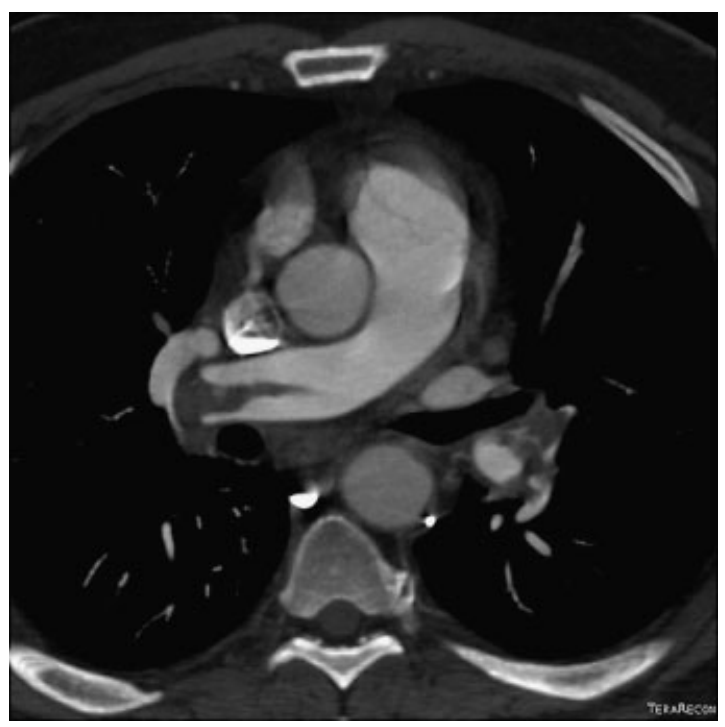

Fig. 1 Extensive bilateral pulmonary emboli are demonstrated by computed tomographic arteriography (CTA). The patient was hemodynamically stable but was tachycardic (heart rate 130 beats/ min) and required $60 \%$ oxygen by facemask. The troponin was positive, and brain natriuretic peptide was elevated to three times the upper limits of normal. CTA revealed significant right ventricular enlargement. This was also noted by echocardiography, and there was marked right ventricular hypokinesis as well. Based on these features in this patient with submassive PE, systemic thrombolysis was administered with marked improvement and without complications. Intravenous heparin was continued during the 2-hour tPA infusion. 


\section{Which Agent or Protocol Should Be Used?}

There are no head-to-head trials indicating that a particular thrombolytic agent has superior efficacy or safety; studies have generally compared thrombolysis to anticoagulation alone. ${ }^{15,17,19,30}$ Recombinant tPA (alteplase), streptokinase, and recombinant human urokinase are the best studied thrombolytic agents for the treatment of acute PE. Streptokinase is the least expensive but the most commonly associated with adverse effects, including allergic reactions and hypotension.

Newer agents approved for acute coronary syndromes such as tenecteplase and reteplase have not been approved for use in acute PE but have been studied. ${ }^{15,17,32}$ In the Italian Tenecteplase Italian Pulmonary Embolism Study trial, the effect of tenecteplase versus anticoagulation on RV dysfunction assessed by echocardiography in hemodynamically stable patients with PE was evaluated in a multicenter, randomized, double-blind, placebo-controlled study. ${ }^{32} \mathrm{RV}$ dysfunction was defined as a right/left ventricle end-diastolic dimension ratio $>1$ in the apical four-chamber view, and reduction in RV dysfunction at 24 hours was the primary efficacy endpoint. The results suggested that in hemodynamically stable patients with $\mathrm{PE}$, treatment with single-bolus tenecteplase is feasible at the same doses used for acute myocardial infarction and is associated with reduction of RV dysfunction at 24 hours. Whether this benefit is associated with an improved clinical outcome without excessive bleeding is being explored with tenecteplase in the much larger PEITHO trial. ${ }^{31}$

Available evidence suggests that shorter infusions (i.e., $\leq 2$ hours) achieve more rapid clot lysis and are associated with lower rates of bleeding than longer ones (i.e., 12 hours). ${ }^{13}$ Thus, of the approved agents, tPA has been recommended due to its short infusion time. ${ }^{13,14}$ In the PEITHO trial, the tenecteplase is delivered by an even faster bolus ( 5 to 10 seconds). ${ }^{31}$

Although it has been advised that anticoagulation be discontinued during the thrombolytic infusion and restarted after the infusion when the activated partial thromboplastin time is 80 seconds or less no data support the need to stop anticoagulation. However, in many non-US countries, the anticoagulation infusion is continued during thrombolytic therapy. When bleeding risk is deemed higher, standard unfractionated heparin may be preferred based on its short action and reversibility. No randomized trial data support this approach either. The Assessment of the Safety and Efficacy of New Thrombolytic Regimens 3 study examined different anticoagulation regimens in acute myocardial infarction patients receiving tenecteplase. ${ }^{33}$ The conclusion was that enoxaparin was safe and superior to standard heparin in this setting. Nonetheless, in acute PE for which thrombolytics are administered, there is no clear standard of care in this regard.

\section{Complications of Thrombolytic Therapy}

Because thrombolytic agents are intravenous agents with systemic action, they lyse clots anywhere within the vasculature. Thus, complications from bleeding, with intracerebral hemorrhage being the most feared, become relevant, although the latter is relatively rare when a careful risk assessment has been undertaken (similar to myocardial infarction). In the International Cooperative Pulmonary Embolism Registry, intracranial bleeding occurred in 3.0\% of the 304 patients who received thrombolytic therapy, compared with only $0.3 \%$ in the placebo group, suggesting that the risk is not only increased but also that the "real-life" risk may be higher than in randomized clinical trials. ${ }^{16}$

Several absolute and relative contraindications to thrombolytic therapy have been proposed to minimize the bleeding risk (-Table 2). However, in extreme clinical circumstances, even absolute contraindications may not preclude the use of thrombolytics in the eyes of some clinicians. Pooled data from available randomized trials of thrombolytics for acute $\mathrm{PE}^{1-11}$ have shown a trend toward increased major bleeding events in the thrombolytic group versus the group that received heparin alone; however, this does not reach statistical significance (9.1 vs. $6.1 \%$; odds ratio, $0.67 ; 95 \% \mathrm{CI}, 0.40$ to 1.12 ). However, minor bleeding events were significantly increased in the thrombolytic group ( 22.7 vs. $10 \%$; odds ratio, 2.63 ; $95 \%$ CI, 1.53 to 4.54$).^{19}$

When systemic thrombolytic therapy is contraindicated, other approaches can be considered. These include catheterbased embolectomy and surgical embolectomy. The precise roles of these techniques have not been precisely defined, however.

Table 2 Absolute and relative contraindications to thrombolytic therapy

\footnotetext{
Absolute contraindications $^{\mathrm{a}}$

- History of intracranial hemorrhage

- Known intracranial neoplasm, arteriovenous malformation or aneurysm

- Significant head trauma

- Active internal bleeding

- Known bleeding diathesis

- Intracerebral or intraspinal surgery within 3 months

- Cerebrovascular accident within 2 months

Relative contraindications

- Recent internal bleeding

- Recent surgery or organ biopsy

- Recent trauma, including cardiopulmonary resuscitation

- Venipuncture at noncompressible site

- Uncontrolled hypertension

- Diabetic retinopathy

- Pregnancy

- Age $>75$ years

${ }^{a}$ Although absolute contraindications should be carefully assessed, some of these (except concurrent intracranial hemorrhage) might not be "absolute" in the most extreme circumstances of massive pulmonary embolism with hemodynamic compromise. (The decision to use thrombolytic therapy depends on the clinician's assessment of PE severity, prognosis, and risk of bleeding). ${ }^{13}$
} 


\section{Surgical Pulmonary Embolectomy and Catheter-Based Techniques}

According to the data of Nationwide Inpatient Sample register from 1998 to 2008, 72,230 patients presented with unstable PE. Of these, only $1.2 \%$ underwent open pulmonary embolectomy and $0.3 \%$ received catheter-tip embolectomy. ${ }^{34}$ With accumulating experience with newer catheter devices communicating favorable outcomes (albeit without randomized trial data) and the concerns of adverse effects of systemic thrombolysis, there has been more interest in these interventions. $^{35}$

There are certain clinical settings where the local management of emboli presents as an appealing alternative; these include massive PE in patients with formal contraindications to thrombolytics, less severe presentations with RV dysfunction, and, finally, as an escalation therapy when systemic thrombolysis has failed.

\section{Surgical Embolectomy}

Unfortunately, surgical embolectomy has been associated with high mortality rates $(\sim 27 \%)$, and this concerns some clinicians. ${ }^{34}$ A substantial contribution to this high mortality is the critically ill nature of these patients. A few centers have liberalized their criteria for acute embolectomy and have operated on patients with preserved systemic blood pressure presenting with extensive clot burdens and concomitant RV dysfunction. ${ }^{36}$ A series of 47 consecutive patients, meeting such criteria, who underwent surgical pulmonary embolectomy (requiring cardiopulmonary bypass but under normothermic conditions and avoiding cardioplegic arrest) showed a survival rate of $96 \%$ at 27 months of follow-up. This high rate of survival was attributed to the multidisciplinary approach, rapid diagnosis (including risk stratification), and, probably most important, improved and immediate surgical technique. ${ }^{36}$

\section{Catheter-Based Techniques}

Modern catheter-based techniques include mechanical fragmentation and/or aspiration of emboli (including rheolytic thrombectomy), often with intraembolic thrombolytic injection.
The latter "pharmacomechanical thrombolysis" technique exposes a greater embolic surface area to the drug's effect. ${ }^{37}$ Simple thrombolytic infusion into the pulmonary artery proximal to the embolus appears to be no more efficacious than systemic delivery. ${ }^{35,38}$

Although various catheter-based devices exist, only minimal evidence-based data support each of them; most of the literature consists of observational studies. Early studies proved that a simple vacuum suction technique could be effective, and there has been a recent resurgence of interest in this approach. ${ }^{39}$ A commonly used method is the rotating pigtail fragmentation catheter, which usually needs adjunctive aspiration due to distal clot embolization. ${ }^{35,37}$ Another technique is the AngioJet rheolytic device (Possis Medical, MN) that uses mechanical thrombolysis and concomitant thrombolytic injection. ${ }^{40,41}$ Hemolysis may occur with this technique. The use of ultrasound to enhance thrombolytic permeation of large emboli has been successfully used. The EKOS catheter (EKOS Corporation, Bothell, WA) is one of the few catheter-based techniques being studied in both retrospective nonrandomized and prospective randomized clinical trials. ${ }^{42,43}$

A novel and promising approach is the AngioVac aspiration system (AngioDynamics, Latham, NY) that is composed of an extracorporeal bypass circuit that facilitates drainage, filtration, and reinfusion of blood cleared from unwanted clot material. ${ }^{44}$ Already approved by the U.S. Food and Drug Administration, this technique appears to have promise as an aggressive technique to treat very large emboli, although few data are published to date. - Table 3 offers a list of catheter-based techniques that have been reported.

Based on the limited data available regarding the effectiveness of each therapy, the choice of surgical or catheterbased embolectomy depends on the availability of resources and institution's expertise.

\section{Conclusions}

Substantial progress in technology and clinical research methods have led to advances in the diagnosis, treatment,

Table 3 Catheter-based embolectomy: Available techniques

\begin{tabular}{|c|c|c|}
\hline Technique & Examples & Manufacturers \\
\hline Aspiration & Greenfield suction catheter & Boston Scientific, Watertown, MA \\
\hline Local thrombolysis $^{\mathrm{a}}$ & tPA (alteplase) & Genentech (Roche), Switzerland \\
\hline Fragmentation & Rotatable pigtail catheter & Cook Europe, The Netherlands \\
\hline \multirow[t]{5}{*}{ Mechanical rheolysis } & Amplatz device & Bard-Microvena, White Bear Lake, MN \\
\hline & Aspirex device $^{\mathrm{b}}$ & Straub Medical, Wangs, Switzerland \\
\hline & Hydrolyser $^{b}$ & Cordis, Warren, NJ \\
\hline & Angiojet ${ }^{b}$ & Possis, Minneapolis, MN \\
\hline & Oasis device & Boston Scientific \\
\hline \multirow[t]{2}{*}{ Angioplasty/stenting } & Wallstent & Schneider Europe AG, Bülach, Switzerland \\
\hline & Gianturco Z stents & Cook Europe, Bjaerskov, Denmark \\
\hline
\end{tabular}

${ }^{a}$ More than 100 cases reported.

${ }^{\mathrm{b}}$ More than 20 cases reported. 
and prevention of acute venous thromboembolism over the past several decades. Although guidelines are useful, they are limited by the existing evidence-based literature so that controversies remain with regard to when and how to administer thrombolytic therapy.

The approach to submassive PE is probably the most controversial area in the field of venous thromboembolism and one in which additional data are clearly needed. Meanwhile, there should be strong consideration for aggressive therapy in certain "hemodynamically stable" patients, such as when RV size and function, biomarkers, clot burden (lungs and legs), and cardiovascular reserve suggest the potential for a high mortality. No clear submassive PE subtype indicates the clear need for therapy beyond anticoagulation, but the higher the clot burden, the more abnormal the RV (particularly in the presence of a positive troponin), and the poorer the oxygenation, the lower the threshold should be for proceeding with an aggressive approach.

With regard to catheter-based embolectomy procedures, it is still impossible to clearly specify precise recommendations for use. It is also not possible to determine superiority of a particular technique due to the lack of comparative and randomized trial data. However, it appears reasonable to consider one of these procedures in patients with proven massive PE and hemodynamic instability, especially when thrombolytic therapy has failed or is contraindicated, as well as in submassive PE patients deemed at high risk for poor outcome by the evaluating clinician. Many clinicians have concerns that aggressive approaches to acute PE are underused. ${ }^{45,46}$ More clinical trials should be conducted.

\section{Disclosures}

No active relevant financial disclosures. Research grants over the past 3 years include Bayer. Consulting over the past 3 years with Sanofi Aventis, Janssen, Boehringer Ingelheim, and EKOS. Lectures over the past 3 years with Sanofi Aventis, EKOS. No lectures for honoraria since 2011.

\section{References}

1 The Urokinase Pulmonary Embolism Trial. A national cooperative study. Circulation 1973;47(2)(Suppl):II1-II108

2 Tibbutt DA, Davies JA, Anderson JA, et al. Comparison by controlled clinical trial of streptokinase and heparin in treatment of lifethreatening pulmonay embolism. BMJ 1974;1(5904):343-347

3 Ly B, Arnesen H, Eie H, Hol R. A controlled clinical trial of streptokinase and heparin in the treatment of major pulmonary embolism. Acta Med Scand 1978;203(6):465-470

4 Dotter CT, Seamon AJ, Rosch J, et al. Streptokinase and heparin in the treatment of pulmonary embolism: a randomized clinical trial. Vasc Surg 1979;13:42-52

5 Marini C, Di Ricco G, Rossi G, Rindi M, Palla R, Giuntini C. Fibrinolytic effects of urokinase and heparin in acute pulmonary embolism: a randomized clinical trial. Respiration 1988;54(3): 162-173

6 Levine M, Hirsh J, Weitz J, et al. A randomized trial of a single bolus dosage regimen of recombinant tissue plasminogen activator in patients with acute pulmonary embolism. Chest 1990;98(6): 1473-1479

7 The PIOPED Investigators. Tissue plasminogen activator for the treatment of acute pulmonary embolism. A collaborative study by the PIOPED Investigators. Chest 1990;97(3):528-533

8 Dalla-Volta S, Palla A, Santolicandro A, et al. PAIMS 2: alteplase combined with heparin versus heparin in the treatment of acute pulmonary embolism. Plasminogen activator Italian multicenter study 2. J Am Coll Cardiol 1992;20(3):520-526

9 Goldhaber SZ, Haire WD, Feldstein ML, et al. Alteplase versus heparin in acute pulmonary embolism: randomised trial assessing right-ventricular function and pulmonary perfusion. Lancet 1993; 341(8844):507-511

10 Jerjes-Sanchez C, Ramírez-Rivera A, Arriaga-Nava R, et al; de Lourdes García M. Streptokinase and heparin versus heparin alone in massive pulmonary embolism: a randomized controlled trial. J Thromb Thrombolysis 1995;2(3):227-229

11 Konstantinides S, Geibel A, Heusel G, Heinrich F, Kasper W; Management Strategies and Prognosis of Pulmonary Embolism3 Trial Investigators. Heparin plus alteplase compared with heparin alone in patients with submassive pulmonary embolism. $\mathrm{N}$ Engl J Med 2002;347(15):1143-1150

12 Indications for fibrinolytic therapy in suspected acute myocardial infarction: collaborative overview of early mortality and major morbidity results from all randomised trials of more than 1000 patients. Fibrinolytic Therapy Trialists' (FTT) Collaborative Group. Lancet 1994;343(8893):311-322

13 Kearon C, Akl EA, Comerota AJ, et al. Antithrombotic therapy for VTE disease: antithrombotic therapy and prevention of thrombosis. 9th ed. American College of Chest Physicians Evidence-Based Clinical Practice Guidelines. Chest 2012;141:e419S

14 Jaff MR, McMurtry MS, Archer SL, et al; American Heart Association Council on Cardiopulmonary, Critical Care, Perioperative and Resuscitation; American Heart Association Council on Peripheral Vascular Disease; American Heart Association Council on Arteriosclerosis, Thrombosis and Vascular Biology. Management of massive and submassive pulmonary embolism, iliofemoral deep vein thrombosis, and chronic thromboembolic pulmonary hypertension: a scientific statement from the American Heart Association. Circulation 2011;123(16):1788-1830

15 Thabut G, Thabut D, Myers RP, et al. Thrombolytic therapy of pulmonary embolism: a meta-analysis. J Am Coll Cardiol 2002; 40(9):1660-1667

16 Goldhaber SZ, Visani L, De Rosa M. Acute pulmonary embolism: clinical outcomes in the International Cooperative Pulmonary Embolism Registry (ICOPER). Lancet 1999;353(9162):1386-1389

17 Dong B, Jirong Y, Liu G, Wang Q, Wu T. Thrombolytic therapy for pulmonary embolism. Cochrane Database Syst Rev 2006;(2): CD004437

18 Jiménez D, Aujesky D, Moores L, et al; RIETE Investigators. Simplification of the pulmonary embolism severity index for prognostication in patients with acute symptomatic pulmonary embolism. Arch Intern Med 2010;170(15):1383-1389

19 Wan S, Quinlan DJ, Agnelli G, Eikelboom JW. Thrombolysis compared with heparin for the initial treatment of pulmonary embolism: a meta-analysis of the randomized controlled trials. Circulation 2004;110(6):744-749

20 Lu MT, Cai T, Ersoy H, et al. Interval increase in right-left ventricular diameter ratios at $\mathrm{CT}$ as a predictor of 30-day mortality after acute pulmonary embolism: initial experience. Radiology 2008; 246(1):281-287

21 Vedovati MC, Becattini C, Agnelli G, et al. Multidetector computed tomography for acute pulmonary embolism: Embolic burden and clinical outcome. Chest 2012;142(6):1417-1424

22 Kostrubiec M, Pruszczyk P, Bochowicz A, et al. Biomarker-based risk assessment model in acute pulmonary embolism. Eur Heart J 2005;26(20):2166-2172 
23 Becattini C, Vedovati MC, Agnelli G. Prognostic value of troponins in acute pulmonary embolism: a meta-analysis. Circulation 2007 ; 116(4):427-433

24 Cavallazzi R, Nair A, Vasu T, Marik PE. Natriuretic peptides in acute pulmonary embolism: a systematic review. Intensive Care Med 2008;34(12):2147-2156

25 Reichlin T, Hochholzer W, Bassetti S, et al. Early diagnosis of myocardial infarction with sensitive cardiac troponin assays. N Engl J Med 2009;361(9):858-867

26 Lankeit M, Friesen D, Aschoff J, et al. Highly sensitive troponin T assay in normotensive patients with acute pulmonary embolism. Eur Heart J 2010;31(15):1836-1844

27 Lankeit M, Jiménez D, Kostrubiec M, et al. Predictive value of the high-sensitivity troponin $\mathrm{T}$ assay and the simplified Pulmonary Embolism Severity Index in hemodynamically stable patients with acute pulmonary embolism: a prospective validation study. Circulation 2011;124(24):2716-2724

28 Jiménez D, Aujesky D, Moores L, et al. Combinations of prognostic tools for identification of high-risk normotensive patients with acute symptomatic pulmonary embolism. Thorax 2011;66(1): 75-81

29 Jiménez D, et al. Prognostic significance of deep venous thrombosis in patients presenting with acute symptomatic pulmonary embolism. Am J Respir Crit Care Med 2010;18: 983-991

30 Konstantinides S, Tiede N, Geibel A, Olschewski M, Just H, Kasper $W$. Comparison of alteplase versus heparin for resolution of major pulmonary embolism. Am J Cardiol 1998;82(8):966-970

31 PEITHO pulmonary embolism thrombolysis study. Available at: http://clinicaltrials.gov/ct2/show/NCT00639743. Accessed January 12,2013

32 Becattini C, Agnelli G, Salvi A, et al; TIPES Study Group. Bolus tenecteplase for right ventricle dysfunction in hemodynamically stable patients with pulmonary embolism. Thromb Res 2010; 125(3):e82-e86

33 Assessment of the Safety and Efficacy of a New Thrombolytic Regimen (ASSENT)-3 Investigators. Efficacy and safety of tenecteplase in combination with enoxaparin, abciximab, or unfractionated heparin: the ASSENT-3 randomised trial in acute myocardial infarction. Lancet 2001;358(9282):605-613

34 Kilic A, Shah AS, Conte JV, Yuh DD. Nationwide outcomes of surgical embolectomy for acute pulmonary embolism. J Thorac Cardiovasc Surg 2013;145(2):373-377
35 Kuo WT, Gould MK, Louie JD, Rosenberg JK, Sze DY, Hofmann LV. Catheter-directed therapy for the treatment of massive pulmonary embolism: systematic review and meta-analysis of modern techniques. J Vasc Interv Radiol 2009;20(11):1431-1440

36 Leacche M, Unic D, Goldhaber SZ, et al. Modern surgical treatment of massive pulmonary embolism: results in 47 consecutive patients after rapid diagnosis and aggressive surgical approach. J Thorac Cardiovasc Surg 2005;129(5):1018-1023

37 Tapson VF, Gurbel PA, Witty LA, Pieper KS, Stack RS. Pharmacomechanical thrombolysis of experimental pulmonary emboli. Rapid low-dose intraembolic therapy. Chest 1994;106(5): 1558-1562

38 Tapson VF. Interventional therapies for venous thromboembolism: vena caval interruption, surgical embolectomy, and catheterdirected interventions. Clin Chest Med 2010;31(4):771-781

39 Greenfield LJ, Kimmell GO, McCurdy WC III. Transvenous removal of pulmonary emboli by vacuum-cup catheter technique. J Surg Res 1969;9(6):347-352

40 Siablis D, Karnabatidis D, Katsanos K, Kagadis GC, Zabakis P, Hahalis G. AngioJet rheolytic thrombectomy versus local intrapulmonary thrombolysis in massive pulmonary embolism: a retrospective data analysis. J Endovasc Ther 2005;12(2): 206-214

41 Margheri M, Vittori G, Vecchio S, et al. Early and long-term clinical results of AngioJet rheolytic thrombectomy in patients with acute pulmonary embolism. Am J Cardiol 2008;101(2):252-258

42 Engelhardt TC, Taylor AJ, Simprini LA, Kucher N. Catheter-directed ultrasound-accelerated thrombolysis for the treatment of acute pulmonary embolism. Thromb Res 2011;128(2):149-154

43 A prospective, single-arm, multi-center trial of EkoSonic ${ }^{\circledR}$ endovascular system and activase for treatment of acute pulmonary embolism (PE). (SEATTLE II). Available at: http://www.clinicaltrials.gov/ct2/show/NCT01513759. Accessed January 12, 2013

44 Todoran TM, Sobieszczyk PS, Levy MS, et al. Percutaneous extraction of right atrial mass using the Angiovac aspiration system. J Vasc Interv Radiol 2011;22(9):1345-1347

45 Stein PD, Matta F. Thrombolytic therapy in unstable patients with acute pulmonary embolism: saves lives but underused. Am J Med 2012;125(5):465-470

46 Tapson VF. What will it take to initiate a move toward a more aggressive therapeutic approach to venous thromboembolism? Am J Med 2008;121(11)(Suppl 1):S20-S27 